\title{
Energetics of Lipid Bilayers with Applications to Deformations In- duced by Inclusions
}

\author{
Raffaella De Vita, ${ }^{* a}$ and Iain W. Stewart, ${ }^{b}$
}

\author{
Received Xth $X X X X X X X X X X 20 X X$, Accepted Xth $X X X X X X X X X 20 X X$ \\ First published on the web Xth $X X X X X X X X X X 200 X$ \\ DOI: $10.1039 / b 000000 x$
}

A new energy for the description of large deformations of lipid bilayers is formulated with mathematical rigor. This energy is derived by considering the smectic A liquid crystalline nature of lipid bilayers and the coupling between the deformations of the layers and their constituent lipid molecules. Analogies between smectic A liquid crystals, with an infinite number of layers, and lipid bilayers, with a finite number of layers, are further discussed. The novelty of the energy density is demonstrated by studying the large deformations of planar lipid bilayers induced by cylindrical inclusions. The results of this study are directly compared with the results obtained using May's theoretical framework [May, Eur. Biophys. J., 2000, 29, 17-28] in which small deformations are assumed. As expected, the proposed energy density predicts larger distortions of the lipid molecules and deformations of the lipid bilayers close to an inclusion.

\section{Introduction}

Lipid bilayers are currently being used for engineering numerous bioinspired microsystems ranging from portable and fast biosensors for detecting biological agents ${ }^{33,37}$ to biocompatible and biodegradable drug delivery carriers. ${ }^{6,57}$ Many of these micro-systems work as proof of concept in laboratory environments. Their application in real-world scenarios, however, remains to be demonstrated due to the poor stability of lipid bilayers to mechanical disturbances. ${ }^{23}$ Current challenges encountered in experimental biomechanics (e.g., measurement of physical quantities in the nanoscale range) prevent an accurate characterization of the mechanical properties of lipid bilayers, which is needed to enhance their performance. Therefore, the formulation of reliable mathematical models is essential in making a big leap forward in the development of the next generation of bio-inspired microsystems that include lipid bilayers.

There has been extensive research on modeling the physical properties of lipid bilayers by employing molecular dynamics, ${ }^{29}$ coarse-grained models ${ }^{11,51}$ and continuum models. ${ }^{12,60}$ Molecular dynamics simulations are very powerful tools for studying the microstructure of lipid bilayers, especially their interactions with different molecules and proteins. ${ }^{20,68}$ Due to current limitations in computing power, molecular dynamics can only be used for simulating phenomena that occur at the

a Department of Engineering Science and Mechanics, 230 Norris Hall, Blacksburg, Virginia, USA. Fax: 540-231-4574; Tel: 540-231-5905; E-mail: devita@vt.edu

${ }^{b}$ Department of Mathematics and Statistics, University of Strathclyde, Glasgow, Scotland, UK. nano-meter spatial scale and nano-second time scale. ${ }^{11,39}$ In coarse-grained models, some fine details about the structure of lipids are averaged out so that the simulations are computationally less expensive. ${ }^{38,52}$ Continuum models are, however, preferred for simulating physical phenomena over long length and time scales, which are relevant to many processes in cell biology, experimental studies, and real-world applications involving lipid bilayers.

One of the most successful continuum models for lipid bilayers is the spontaneous curvature model first formulated by Canham ${ }^{15}$ and then refined by Helfrich. ${ }^{36}$ According to the spontaneous curvature model, the free energy per unit area, $w$, of lipid bilayers can be expressed in terms of the mean curvature, $H$, and Gaussian curvature, $K$, as

$$
w=\frac{\kappa}{2}\left(2 H-s_{0}\right)^{2}+\bar{\kappa} K,
$$

where $\kappa$ is the curvature or bending modulus, $\bar{\kappa}$ is the saddlesplay or Gaussian curvature modulus, and $s_{0}$ is the spontaneous curvature, which takes into account possible asymmetries between the two leaflets of the lipid bilayer. The mean and Gaussian curvatures can be defined in terms of the principal radii of curvature, $\frac{1}{R_{1}}$ and $\frac{1}{R_{2}}$, or, in a notation more familiar in liquid crystals, in terms of the unit normal to the lipid layers, a (Fig. 1(a)). It can be easily shown that ${ }^{43}$

$$
\begin{gathered}
H=\frac{1}{2}\left(\frac{1}{R_{1}}+\frac{1}{R_{2}}\right)=\frac{1}{2} \nabla \cdot \mathbf{a}, \\
K=\frac{1}{R_{1} R_{2}}=\nabla \cdot((\nabla \cdot \mathbf{a}) \mathbf{a})+\nabla \cdot(\mathbf{a} \times(\nabla \times \mathbf{a})) .
\end{gathered}
$$



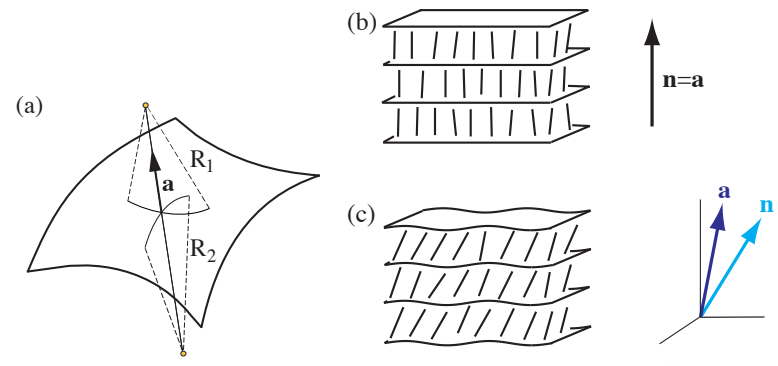

Fig. 1 (a) Curvature for a two-dimensional surface. $R_{1}$ and $R_{2}$ denote the radii of curvature and the vector a represents the normal to the surface. (b-c) The short bold lines represent the molecules while the planes represent the layer alignment of the smectic A liquid crystals. (b) Undistorted smectic A liquid crystal alignment: the director $\mathbf{n}$ coincides with the layer normal a. (c) Distorted smectic A liquid crystal alignment: the director $\mathbf{n}$ decouples from the layer normal a.

The spontaneous curvature model has been derived by Helfrich from the Frank energy density for liquid crystals in which the normal to the layers, a, coincides with the director, $\mathbf{n}$, which defines the alignment of the molecules ${ }^{28,63}$ (Fig. 1(b)). The relationships among the curvature moduli, $\kappa$ and $\bar{\kappa}$, and the constants, $k_{11}, k_{22}$ and $k_{24}$, in the Frank's energy density ${ }^{28}$ are $\kappa=k_{11} d$, and $\bar{\kappa}=-\left(k_{22}+k_{24}\right) d$, where $d$ is the thickness of the membrane. ${ }^{55}$ This model has been used by hundreds of researchers to study various configurations of lipid bilayers including their interactions with channels and inclusions. $1,2,8,9,22,32$ Thus, citing here all the great research that has followed from Canham and Helfrich's pioneering work is clearly impossible.

The spontaneous curvature model cannot describe the tilt of the lipid molecules that is associated with the deformation of the layers. Indeed, the energy per unit area presented in eqs. (1)-(3) depends only on the normal to the layers, a, and does not contain any term that accounts for the tilting of the molecules. As recognized by Helfrich in his seminal work ${ }^{36}$ and, more recently, by other investigators, ${ }^{30,34,47-49}$ such tilt of the lipid molecules should be taken into account when modeling lipid bilayers. The most comprehensive approach, in which different energetic contributions are considered, has been presented by May. ${ }^{47}$ May's analysis is limited to small distortions of the lipid molecules and small displacements of the layers. However, lipid layers undergo large deformations such as those observed experimentally with vesicles ${ }^{61}$ and the tilt of lipid molecules experiences distortion measured to be as large as $40^{\circ} .{ }^{67}$ Thus, in order to accurately characterize the mechanical performance of lipid-based micro-devices, continuum models must be formulated to describe the large distortions of lipid assemblies and decoupling between the normal to the layers, a, and the director alignment, $\mathbf{n}$ (Fig. 1(c)).
The objective of this manuscript is to derive novel continuum models for characterizing the equilibrium configurations of lipid bilayers. The great success of the spontaneous curvature model derived by Helfrich ${ }^{36}$ from the Frank energy density for liquid crystals supports the idea that lipid bilayers must be modeled by accounting for their liquid crystalline nature. The new models will be formulated within the context of a new nonlinear theory for smectic A liquid crystals in which the alignment of the lipid molecules, which is defined by the unit vector $\mathbf{n}$, is not constrained to coincide with the smectic layer normal, which is defined by the unit vector $\mathbf{a}^{64}$ (Fig. 1(c)). This assumption makes the new theory more attractive than the theory for smectic A liquid crystals proposed by de Gennes ${ }^{24}$ and the well-known spontaneous curvature model $^{36}$ used for lipid bilayers.

\section{Energy density for lipid bilayers}

The following general energy density, $w_{D S}$, is proposed for lipid bilayers

$$
\begin{aligned}
& w_{D S}= \frac{1}{2} K_{1}^{a}(\nabla \cdot \mathbf{a})^{2}+\frac{1}{2} K_{1}^{n}\left(\nabla \cdot \mathbf{n}-s_{0}\right)^{2} \\
&+\frac{1}{2} K_{2} \nabla \cdot[(\mathbf{n} \cdot \nabla) \mathbf{n}-(\nabla \cdot \mathbf{n}) \mathbf{n}]+\frac{1}{2} B_{0}|\nabla \Phi|^{-2}(1-|\nabla \Phi|)^{2} \\
& \quad+\frac{1}{2} B_{1}\left[1-(\mathbf{n} \cdot \mathbf{a})^{2}\right]+B_{2}(\nabla \cdot \mathbf{n})\left(1-|\nabla \Phi|^{-1}\right),
\end{aligned}
$$

where $K_{1}^{a}, K_{1}^{n}, K_{2}, B_{0}, B_{1}$, and $B_{2}$ are material constants. The first term on the right-hand side is the bending energy, the second term is the splay energy with $s_{0}$ denoting the spontaneous splay, the third term is the saddle-splay energy, the fourth term is the compression-expansion energy, the fifth term is the energy due to the coupling between $\mathbf{n}$ and $\mathbf{a}$, and the sixth term is the energy due to the coupling between the splay and compression-expansion of the layer.

The scalar function $\Phi$ in eq. (4) defines the layer structure of a smectic A liquid crystal in a sense that will be described in detail in Sec. 3. The unit normal to the smectic layer, $\mathbf{a}$, is derived from it by setting

$$
\mathbf{a}=\nabla \Phi /|\nabla \Phi| .
$$

For example, $\Phi=z$ and $\mathbf{a}=(0,0,1)$ for planar smectic layers that are parallel to the $x y$ plane in the usual Cartesian description.

The energy density $w_{D S}$ presented in eq. (4) needs to be nonnegative. Clearly, this requires that the material constants $K_{1}^{a}$, $K_{1}^{n}, B_{0}$, and $B_{1}$ be non-negative, since these are the coefficients of quadratic terms. In order to determine a priori restrictions on $K_{2}$ and $B_{2}$ in relation to the other material constants, we can follow standard methods used in liquid crystal theory. ${ }^{63}$ Thus, we first note that because $K_{1}^{a}$ and $B_{1}$ are non-negative and their 
related expressions $\nabla \cdot \mathbf{a}$ and $\mathbf{n} \cdot \mathbf{a}$ do not occur elsewhere in eq. (4), the remaining terms in such equation can be written as a quadratic form that only involves the material constants $K_{1}^{n}$, $K_{2}, B_{0}$, and $B_{2}$. Moreover, the following identity ${ }^{63}$

$$
\nabla \cdot[(\mathbf{n} \cdot \nabla) \mathbf{n}-(\nabla \cdot \mathbf{n}) \mathbf{n}]=\operatorname{tr}\left((\nabla \mathbf{n})^{2}\right)-(\nabla \cdot \mathbf{n})^{2}
$$

enables the required quadratic form for the remaining terms to be written as $\frac{1}{2} \mathbf{v}^{\mathrm{T}} \cdot \mathbf{A} \cdot \mathbf{v}$ where, for $s_{0}=0$, $\mathbf{v}$ is a $3 \times 1$-matrix and $\mathbf{A}$ is a $3 \times 3$-matrix defined by

$$
\begin{aligned}
\mathbf{v}=\left(\begin{array}{c}
\nabla \cdot \mathbf{n} \\
\sqrt{\operatorname{tr}\left((\nabla \mathbf{n})^{2}\right)} \\
1-|\nabla \Phi|^{-1}
\end{array}\right), \\
\mathbf{A}=\left(\begin{array}{ccc}
K_{1}^{n}-K_{2} & 0 & B_{2} \\
0 & K_{2} & 0 \\
B_{2} & 0 & B_{0}
\end{array}\right)
\end{aligned}
$$

while, for $s_{0} \neq 0, \mathbf{v}$ is a $4 \times 1$-matrix and $\mathbf{A}$ is a $4 \times 4$-matrix defined by

$$
\begin{array}{r}
\mathbf{v}=\left(\begin{array}{c}
\nabla \cdot \mathbf{n} \\
s_{0} \\
\sqrt{\operatorname{tr}\left((\nabla \mathbf{n})^{2}\right)} \\
1-|\nabla \Phi|^{-1}
\end{array}\right), \\
\mathbf{A}=\left(\begin{array}{cccc}
K_{1}^{n}-K_{2} & -K_{1}^{n} & 0 & B_{2} \\
-K_{1}^{n} & K_{1}^{n} & 0 & 0 \\
0 & 0 & K_{2} & 0 \\
B_{2} & 0 & 0 & B_{0}
\end{array}\right) .
\end{array}
$$

The quadratic form $\frac{1}{2} \mathbf{v}^{\mathrm{T}} \cdot \mathbf{A} \cdot \mathbf{v}$ is positive semi-definite if and only if the determinants of all the principal submatrices of the symmetric matrix $\mathbf{A}$ are non-negative. ${ }^{50}$ It is a simple exercise to show that, for $s_{0}=0$, the material constants need to satisfy the inequalities

$$
\begin{array}{r}
K_{1}^{a} \geq 0, \quad K_{1}^{n} \geq K_{2}, \quad K_{2} \geq 0, \quad B_{0} \geq 0, \quad B_{1} \geq 0, \\
\left(K_{1}^{n}-K_{2}\right) B_{0}-B_{2}^{2} \geq 0,
\end{array}
$$

while, for $s_{0} \neq 0$, the material constants must satisfy the inequalities

$$
\begin{aligned}
K_{1}^{a} \geq 0, \quad K_{1}^{n} \geq 0, \quad K_{2}=0, \quad B_{0} \geq 0, \quad & B_{1} \geq 0, \\
& B_{2}=0
\end{aligned}
$$

It must be noted that the inequalities that arise when $s_{0}=0$ imply that when $K_{1}^{n}=K_{2}$ then $B_{2}=0$.

The energy density $w_{D S}$ in eq. (4) does not rule out the possibility that $\mathbf{n}$ and a may coincide at specific locations. It is invariant under the changes in sign $\mathbf{a} \rightarrow-\mathbf{a}$ or, equivalently, $\nabla \Phi \rightarrow-\nabla \Phi$. If we require $w$ to be invariant to a change in sign of the director $\mathbf{n}$ then $s_{0}$ must be set to zero and $B_{2}=0$ : this is generally not necessarily the case for lipid bilayers in which $\mathbf{n}$ and $-\mathbf{n}$ are distinguishable. With the exception of the $K_{1}^{a}$-term, we will show in Sec. 4 that this energy density when linearized is the same as the one proposed by May ${ }^{47}$ to model the deformation of a planar lipid bilayer induced by an inclusion.

\section{Compression Energy Term}

\subsection{Discrete Case}

The energy density presented in eq. (4) can be used to model both smectic A liquid crystals, with an infinite number of layers, and lamellar structures, with a finite number of layers, such as lipid bilayers. Of course, in general liquid crystals the energy density is required to be invariant to the change in sign of the director $\mathbf{n}$ and $s_{0}$ and $B_{2}$ need to be set to zero. These requirements are absent when modeling lipid bilayers. It will be shown hereafter that the compression-expansion energy term, namely the $B_{0}$-term in eq. (4), has the same form for smectic A liquid crystals as it has for discrete numbers of lamellae. Toward this end, we will adopt many of the mathematical arguments employed by Capriz, ${ }^{13,14}$ Napoli ${ }^{53,54}$ and Capriz and Napoli. ${ }^{16}$ We will use the terminology of smectic liquid crystals from the outset and, in the discourse that follows in the next section, draw upon the parallels with the theory of lipid bilayers.

The lamellar structure of smectic A liquid crystals arises from a periodic mass density distribution that they exhibit. It is well known that the corresponding smectic order can be characterized by a periodic mass density, $\rho$, having the form of a Fourier series that can be approximated by $18,24,42,59$

$$
\rho(\mathbf{x}) \approx \rho_{0}+\rho_{1} \cos \left(2 \pi \Phi(\mathbf{x}, t) / d_{*}\right),
$$

where $\mathbf{x}=\mathbf{x}(t)$ is the position vector of a generic point in space at any time $t, \rho_{0}$ and $\rho_{1}$ are constant amplitudes, $d_{*}$ is the constant common interlayer spacing of the smectic A liquid crystals in the initial configuration. The time variable, $t$, is here introduced to describe distinct configurations of the lamellar structure. The initial configuration is taken to be the one in which all the layers are equidistant. The smectic layers can therefore be represented by $k$ surfaces, $\Sigma_{k}$ for $k=0,1,2,3 \ldots$, which can be defined at any given time $t$ as the level sets of $\Phi(\mathbf{x}, t)$ through the relation

$$
\Phi(\mathbf{x}, t)=k d_{*}
$$

For example, a planar layered sample of smectic A, whose layers are normal to the $z$-axis, can be represented by $z=k d_{*}$ at a fixed time. At any other different time, away from any planar layered configuration, the layer structure will continue to be the level set of $\Phi(\mathbf{x}, t)=k d_{*}$. However, such an equation need 


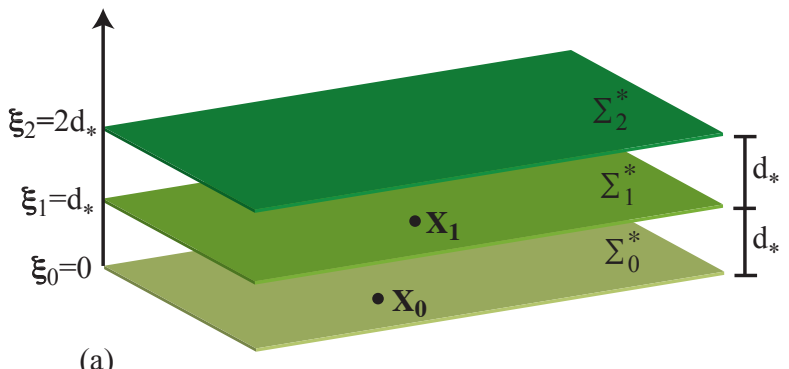

(a)

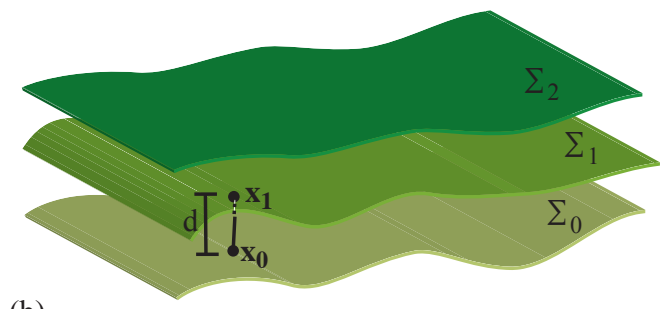

(b)

Fig. 2 Lamellae in reference configuration at $t=0$ (a) and current configuration at $t>0(\mathrm{~b})$.

not necessarily lead to a description of a planar layered configuration, despite the mass density of the sample being the same. The surfaces $\Sigma_{k}$ defined by $\Phi(\mathbf{x}, t)=k d_{*}$ are, therefore, monitoring the points in space of equal mass density as the time $t$ evolves. These surfaces are the two dimensional analogues of the very familiar one-dimensional contours, the isobars, which appear on two-dimensional air pressure charts. The contours on such charts represent the locations of air at certain fixed pre-assigned magnitudes of pressure and these contours can evolve on the chart with time, yet they always represent the originally assigned values of the air pressure. Similarly, the level sets defined by eq. (12) represent the positions in space of the smectic layers where the mass density is concentrated and these surfaces can analogously evolve and change position as time progresses. We remark that the smectic layers, defined by level sets, need not remain equidistant, as pointed out by Maxwell ${ }^{46}$ in his pioneering work on level surfaces.

Consider the lamellar system represented in Fig. 2. It proves convenient to take an initial configuration at $t=0$ that consists of layers that are delimited by equidistant surfaces denoted by $\Sigma_{k}^{*}$ where $k=0,1,2, \ldots$, as shown in Fig. 2(a). If we set $\xi_{k}=k d_{*}$ where $d_{*}$ is the previously defined common distance between two adjacent surfaces, then such surfaces are defined as the level sets of $\Phi^{*}(\mathbf{X})=\xi_{k}, k=0,1,2,3, \ldots$, with

$$
\Phi^{*}(\mathbf{X}) \equiv \Phi(\mathbf{x}(0), 0)=\mathbf{a}_{*} \cdot \mathbf{X}=\xi_{k}
$$

where $\mathbf{a}_{*}$ is a constant vector normal to the surfaces and $\mathbf{X}=$ $\mathbf{x}(0)$ is the position of a point at $t=0$ on the $k$ th delimiting surface $\Sigma_{k}^{*}$.

Next, consider the distortion of the lamellar system at some fixed time $t>0$ depicted in Fig. 2(b). Let $\mathbf{x}_{0}$ be an arbitrary fixed point on the distorted surface $\Sigma_{0}, \mathbf{x}_{0} \in \Sigma_{0}$. The shortest distance from $\mathbf{x}_{0}$ to the distorted surface $\Sigma_{1}$, which is denoted by $d$, must be taken along a direction perpendicular to the surface $\Sigma_{1}$ at some point $\mathbf{x}_{1} \in \Sigma_{1}$, as shown in Fig. 2(b) (which may or may not be unique). This direction is given by the unit vector normal to $\Sigma_{1}$ evaluated at $\mathbf{x}_{1}$ (for definiteness, we will take as the positive direction for this normal to be the one directed towards surface $\Sigma_{1}$ from $\Sigma_{0}$, that is, along the direction of the surfaces $\Sigma_{k}$ as their index $k$ increases). In other words, there exists $d$ such that the vector distance between the two points $\mathbf{x}_{0}$ and $\mathbf{x}_{1}$ at a fixed time $t$, which belong to the level surfaces defined by $\Phi\left(\mathbf{x}_{0}, t\right)=\xi_{0}$ and $\Phi\left(\mathbf{x}_{1}, t\right)=\xi_{1}$, respectively, can be written as

$$
\mathbf{x}_{1}-\mathbf{x}_{0}=d \frac{\nabla \Phi\left(\mathbf{x}_{1}\right)}{\left|\nabla \Phi\left(\mathbf{x}_{1}\right)\right|}
$$

where, for notational brevity, we have suppressed the dependence on the fixed value $t$. It then follows from a Taylor expansion that ${ }^{70}$

$$
\Phi\left(\mathbf{x}_{0}\right)=\Phi\left(\mathbf{x}_{1}-\mathbf{h}\right)=\Phi\left(\mathbf{x}_{1}\right)-\mathbf{h} \cdot \nabla \Phi\left(\mathbf{x}_{1}\right)+R\left(\mathbf{x}_{1}, \mathbf{h}\right),
$$

where

$$
\mathbf{h}=d \frac{\nabla \Phi\left(\mathbf{x}_{1}\right)}{\left|\nabla \Phi\left(\mathbf{x}_{1}\right)\right|}, \quad\left|R\left(\mathbf{x}_{1}, \mathbf{h}\right)\right| /\|\mathbf{h}\| \rightarrow 0 \quad \text { as } \quad\|\mathbf{h}\| \rightarrow 0
$$

with \|\| denoting the Euclidean norm. In this case, $\|\mathbf{h}\|=d$ and hence whenever $d \ll 1$ (for example, if the interlayer spacing is small compared to the lateral dimensions of the smectic layer surface) it is seen that

$$
d_{*}=\xi_{1}-\xi_{0}=\Phi\left(\mathbf{x}_{1}\right)-\Phi\left(\mathbf{x}_{0}\right) \doteqdot d \frac{\nabla \Phi\left(\mathbf{x}_{1}\right)}{\left|\nabla \Phi\left(\mathbf{x}_{1}\right)\right|} \cdot \nabla \Phi\left(\mathbf{x}_{1}\right) .
$$

It follows immediately that

$$
d_{*} \doteqdot d\left|\nabla \Phi\left(\mathbf{x}_{1}\right)\right|
$$

and therefore, for small interlayer distances, we have the relation

$$
\frac{d}{d_{*}}=|\nabla \Phi|^{-1}
$$

where the dependence of $\nabla \Phi$ on the fixed values $\mathbf{x}_{1}$ and $t$ has been omitted for ease of notation. Thus $|\nabla \Phi|^{-1}$ represents the current local interlayer distance measured in units of the reference interlayer distance $d_{*}$. It follows from eq. (19) that there is a compression of the lamellar structure (relative to the initial configuration) when $|\nabla \Phi|>1$ and a dilation when $|\nabla \Phi|<1$. 
We remark that the ratio $d / d_{*}$ need not be close to unity, despite $d$ and $d_{*}$ being small, and that consequently $|\nabla \Phi|$ need not be close to unity.

The relative extension or compression in the local interlayer spacing, $\varepsilon$, which is namely the strain, can be written in a standard form as

$$
\varepsilon=1-\frac{d}{d_{*}}=1-|\nabla \Phi|^{-1} .
$$

The corresponding energy per unit area of $\Sigma_{1}, w_{1}$, is given by

$$
w_{1}=\frac{1}{2} \kappa\left(1-\frac{d}{d_{*}}\right)^{2}=\frac{1}{2} \kappa|\nabla \Phi|^{-2}(1-|\nabla \Phi|)^{2},
$$

where $\kappa\left(\mathrm{N} \mathrm{m}^{-1}\right)$ is analogous to the stiffness constant for one-dimensional solids and $\nabla \Phi$ is evaluated at $\mathbf{x}_{1}$ and a fixed time $t$. Thus the interlayer spacing behaves as a linear elastic spring with stiffness $\kappa$.

In the above arguments, $\mathbf{x}_{0}$ has been chosen arbitrarily in $\Sigma_{0}$ and served to define $x_{1} \in \Sigma_{1}$. It follows that the complete energy, $W_{1}$, for one single layer with delimiting surfaces $\Sigma_{0}$ and $\Sigma_{1}$ is the integral of $w_{1}$ over the surface $\Sigma_{1}$, that is,

$$
W_{1}(t)=\frac{1}{2} \kappa \int_{\Sigma_{1}}|\nabla \Phi(\mathbf{x}, t)|^{-2}(1-|\nabla \Phi(\mathbf{x}, t)|)^{2} d \Sigma_{1},
$$

where $\mathbf{x} \in \Sigma_{1}$. The energy presented in eq. (21) has a clear analogue when extending it to the surface $\Sigma_{2}$. Thus, the energies for two smectic A layers such as a lipid bilayer can be obtained by integrating eq. (21) appropriately over the two surfaces $\Sigma_{1}$ and $\Sigma_{2}$ and adding the results. It is easily seen that the complete energy for a lamellar system of $n$-layers, with $n \geq 2$, is then generally given by

$$
\begin{aligned}
W_{n}(t) & =\sum_{i=1}^{n} \int_{\Sigma_{i}} w_{i} d \Sigma_{i}= \\
& \frac{1}{2} \kappa \sum_{i=1}^{n} \int_{\Sigma_{i}}\left|\nabla \Phi\left(\mathbf{x}_{i}, t\right)\right|^{-2}\left(1-\left|\nabla \Phi\left(\mathbf{x}_{i}, t\right)\right|\right)^{2} d \Sigma_{i},
\end{aligned}
$$

with $\mathbf{x}_{i} \in \Sigma_{i}$. Note that $\nabla \Phi$ may be quite different on distinct layers.

The stiffness $\kappa$ can be estimated for lipid bilayers by using an analogy with smectic liquid crystals. Following the methodology outlined elsewhere ${ }^{55}$ for example, we may choose to set

$$
\kappa=B_{0} d_{*},
$$

as an approximation to $\kappa$ when constructing the energy density contribution for a single layer. Here, $B_{0}$ is the well known compression constant that arises in smectic A liquid crystals.

\subsection{Continuum Case}

When modeling arbitrarily many layers in smectic A liquid crystals, we observe, as pointed out by Capriz, ${ }^{13}$ that the number of layers in a sample is often so great that the interlayer spacing can be considered extremely small in relation to the combined thickness of the smectic A sample. Thus, while for a discrete numbers of layers the function $\Phi$ in eq. (12) is indexed by the discrete values $\xi_{k}$, for an infinite number of layers such a function can be indexed by the continuos real variable $\xi$. Then, eq. (12) can be replaced by

$$
\Phi(\mathbf{x}, t)=\xi,
$$

where we also include dependence on the time $t \geq 0$. The time $t$ can be fixed for the discussion that follows so that the layer structure at any arbitrary time is considered. For notational brevity, we will suppress the dependence on the fixed value $t$. The level sets defined by eq. (25) then describe the smectic surfaces, denoted by $\Sigma_{\xi}$ and indexed over the continuous variable $\xi$ at some given time $t$. It follows that the unit normal to each of these surfaces is given by

$$
\mathbf{a}=\frac{\nabla \Phi(\mathbf{x})}{|\nabla \Phi(\mathbf{x})|}
$$

where $\mathbf{a}$ is evaluated at $\mathbf{x} \in \Sigma_{\xi}$.

From a modeling perspective, it proves convenient to let $\xi$ have the dimension of length and let it represent the shortest distance of the surface represented by $\Sigma_{\xi}^{*}$ from some fixed origin in an initial configuration at $t=0$. In this initial configuration, the surfaces $\Sigma_{\xi}^{*}$ are equidistant planes defined by

$$
\Phi^{*}(\mathbf{X}) \equiv \Phi(\mathbf{x}(0), 0)=\mathbf{a}^{*} \cdot \mathbf{X}=\xi,
$$

where, as before, $\mathbf{a}^{*}$ is a constant vector normal to the surfaces and $\mathbf{X}=\mathbf{x}(0)$ is the position of a point at $t=0$ on the surface $\Sigma_{\xi}^{*}$. For any given fixed value of $\xi, \Phi^{*}(\mathbf{X})=\xi$ represents one particular planar surface in the initial configuration located such that its shortest distance from the origin is $\xi$. Thus, in order to assign a physically meaningful representation to $\xi$, we can be take it to be multiples of the common initial smectic interlayer spacing $d_{*}$. During any subsequent disturbance to the smectic liquid crystal at $t>0$ it is supposed that $\Phi(\mathbf{x}, t)=\xi$. In other words, for each $\xi$ and $t$ fixed, $\Phi=\xi$ is a level set (with constant value $\xi$ ) which represents the location of one particular smectic layer, following the description introduced above. A non-dimensionalization is possible, as discussed by Capriz, ${ }^{13}$ so that $\xi$ may be replaced by integer values $n \in \mathbb{Z}$. This alternative description, also adopted by Blake and Virga, ${ }^{10}$ will not be pursued here.

Following Capriz, ${ }^{13}$ consider an arbitrary point $\mathbf{x}_{1}$ on the distorted surface $\Sigma_{\xi_{1}}, \mathbf{x}_{1} \in \Sigma_{\xi_{1}}$. Let $\mathbf{x}_{2}$ be the closest point to $\mathbf{x}_{1}$ on the distorted surface $\Sigma_{\xi_{2}}$ defined by $\xi_{2}$. Then there exists 
a scalar increment with the dimension of length, labelled $\Delta \eta$, such that

$$
\mathbf{x}_{2}=\mathbf{x}_{1}+\mathbf{a} \Delta \eta
$$

with a defined by eq. (26) and evaluated at $\mathbf{x}=\mathbf{x}_{2}$. Thus, by setting $\Delta \xi \equiv \xi_{2}-\xi_{1}$, one has that

$$
\Delta \xi=\Phi\left(\mathbf{x}_{2}\right)-\Phi\left(\mathbf{x}_{1}\right)=\Phi\left(\mathbf{x}_{1}+\mathbf{a} \Delta \eta\right)-\Phi\left(\mathbf{x}_{1}\right)
$$

and therefore

$$
\frac{\Delta \xi}{\Delta \eta}=\frac{\Phi\left(\mathbf{x}_{1}+\mathbf{a} \Delta \eta\right)-\Phi\left(\mathbf{x}_{1}\right)}{\Delta \eta}
$$

By taking the limit as $\Delta \eta$ tends to zero, we find that righthand side of eq. (30) tends to the derivative of $\xi$ with respect to $\eta, d \xi / d \eta$ while the left-hand side tends to the directional derivative $\nabla \Phi \cdot \mathbf{a}=|\nabla \Phi|$, evaluated at $\mathbf{x}_{2}$. Hence,

$$
\frac{d \xi}{d \eta}=|\nabla \Phi|
$$

In the above equation, $d \eta / d \xi$ can be interpreted as a measure of the number of smectic layers per original initial interlayer length, and so it is the continuum analogue of $d / d_{*}$ in the discrete case considered earlier. Therefore, a corresponding measure of the compression or extension of the layered medium, analogous to eq. (20), is given by

$$
\varepsilon=1-\frac{d \eta}{d \xi}=1-|\nabla \Phi|^{-1}
$$

Similar to the discrete case, there is a compression of the smectic layer structure when $|\nabla \Phi|>1$ and a dilation when $|\nabla \Phi|<1$.

It is now straightforward to see that the analogue of the energy contribution in eq. (21) is actually, in smectic A liquid crystals, a compression energy per unit volume, $w_{c}$, given by 54

$$
w_{c}=\frac{1}{2} B_{0}\left(1-\frac{d \eta}{d \xi}\right)^{2}=\frac{1}{2} B_{0}|\nabla \Phi|^{-2}(1-|\nabla \Phi|)^{2},
$$

where $B_{0}\left(\mathrm{~N} \mathrm{~m}^{-2}\right)$ is analogous to the Young's modulus for one-dimensional solids and $\nabla \Phi$ is evaluated at $\mathbf{x}_{2}$ and $t$. The total bulk energy, $W_{c}$, in a sample volume $V$ is therefore given by

$$
W_{c}=\int_{V} w_{c} d V=\frac{1}{2} B_{0} \int_{V}|\nabla \Phi|^{-2}(1-|\nabla \Phi|)^{2} d V .
$$

The analogy between a discrete system of lamellae such as lipid bilayers and smectic A liquid crystals is evident when a comparison is made between the total energy expressions in eqs. (23) and (34) via the approximation (24). Indeed, if we take the limit as $n \rightarrow \infty$ with a correspondingly suitable

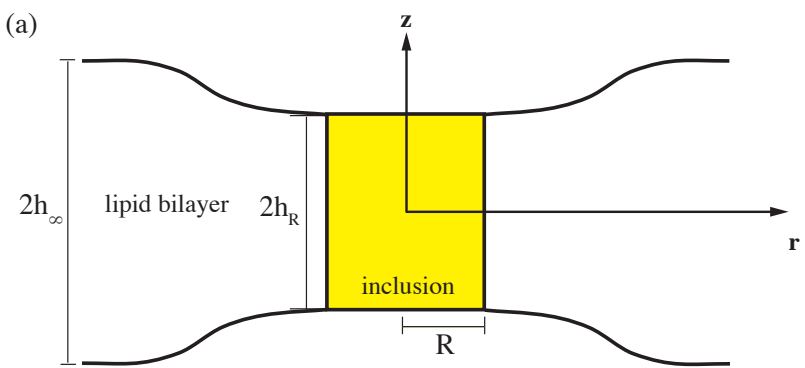

(b)

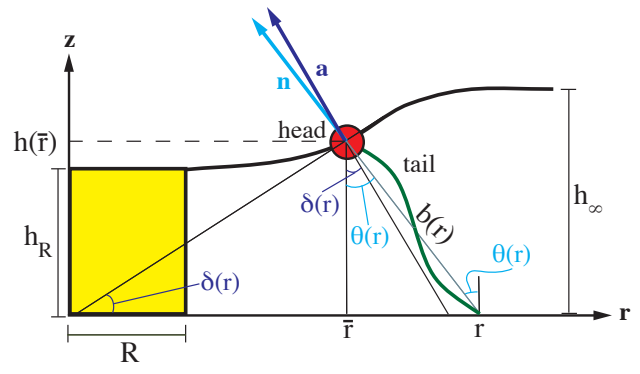

Fig. 3 Cylindrical inclusion in a planar lipid bilayer (a) and its section (b).

simultaneous decrease in the value of $d_{*}$ between layers, then in eq. (23) we see that, approximately,

$$
\kappa \sum_{i=1}^{n} \int_{\Sigma_{i}} w_{i} d \Sigma_{i} \approx B_{0} d_{*} \sum_{i=1}^{n} \int_{\Sigma_{i}} w_{i} d \Sigma_{i} \approx B_{0} \int_{V} w_{c} d V .
$$

Thus there is a direct correspondence between the energy for a discrete numbers of lamellae in eq. (23) and the energy for smectic A liquid crystals in eq. (34).

\section{Applications to Inclusion-induced Deforma- tions}

Consider a planar lipid bilayer with volume $\mathscr{V}$ having a cylindrical inclusion of radius $R$ and height $2 h_{R}$. The lipid bilayer can be assumed to have radial symmetry in the direction of the $\mathbf{r}$-axis about the central $\mathbf{z}$-axis of a cylindrical coordinate system as depicted in Fig. 3. Let us denote by $2 h_{\infty}$ the height of the lipid bilayer far away from the inclusion where $r \rightarrow \infty$ as shown in Fig. 3(a).

Following May's work, ${ }^{47}$ the function $h=h(r)$ defines the height of the planar lipid bilayer, the function $b=b(r)$ defines the length of the lipid molecule, and the variable $\bar{r}$ shown in Fig. 3(b) is defined such that, for $r \geq R$,

$$
\begin{aligned}
& \bar{r}=r-b(r) \sin \theta(r), \\
& h(\bar{r})=b(r) \cos \theta(r),
\end{aligned}
$$


where $\theta=\theta(r)$ is the function that defines the angle formed between the director of the lipid molecules and the $\mathbf{z}$-axis. Let $\delta=\delta(r)$ be the function that defines the angle formed between the normal to the lipid bilayer and the $\mathbf{z}$-axis. It can be shown from a careful consideration of the geometry that

$$
\begin{aligned}
\tan \delta(r)= & \frac{d h(\bar{r})}{d \bar{r}}=\frac{d h(r)}{d r} \frac{d r}{d \bar{r}} \\
& =\frac{b^{\prime}(r) \cos \theta(r)-b(r) \sin \theta(r) \theta^{\prime}(r)}{1-b^{\prime}(r) \sin \theta(r)-b(r) \cos \theta(r) \theta^{\prime}(r)},
\end{aligned}
$$

or, equivalently, that

$$
\begin{aligned}
& \delta(r)= \\
& \quad \arctan \left(\frac{b^{\prime}(r) \cos \theta(r)-b(r) \sin \theta(r) \theta^{\prime}(r)}{1-b^{\prime}(r) \sin \theta(r)-b(r) \cos \theta(r) \theta^{\prime}(r)}\right) .
\end{aligned}
$$

Following our notation, in the coordinate system shown in Fig. 3, the layer normal a that appears in eq. (4) can be written for $r \geq R$ as

$$
\mathbf{a}=-\sin \delta(r) \mathbf{r}+\cos \delta(r) \mathbf{z}
$$

while the director $\mathbf{n}$ for $r \geq R$ has the form

$$
\mathbf{n}=-\sin \theta(r) \mathbf{r}+\cos \theta(r) \mathbf{z}
$$

Given eq. (5), the function $\Phi=\Phi(r, z)$ describing the layer structure can be found by solving the linear partial differential equation defined by

$$
\mathbf{a}=\frac{\nabla \Phi}{|\nabla \Phi|}
$$

Thus, it readily follows that ${ }^{69}$

$$
\begin{aligned}
\frac{\partial \Phi}{\partial r}=-\sin \delta(r), \quad \frac{\partial \Phi}{\partial z}= & \cos \delta(r), \\
& \frac{\partial \Phi}{\partial r}+\tan \delta(r) \frac{\partial \Phi}{\partial z}=0 .
\end{aligned}
$$

By using the methods of characteristics, the solution to eq. (42) for a defined in eq. (40) is found to be

$$
\Phi=c(z-u(r))
$$

where $c$ is a dimensionless constant that can be set to unity. As noted in the previous sections, the surfaces that delimit the lipid layer are defined as the level sets of eq. (44) and are, thus, independent of the value of $c$. The function $u=u(r)$ is the nonlinear displacement of the layer that is equal to

$$
u(r)=u(\infty)-\int_{r}^{\infty} \tan (\delta(t)) d t
$$

where we can assume that $u(\infty)=0$. The height of the planar lipid bilayer defined by the function $h=h(r)$ is related to $u(r)$ via the following

$$
h(r)=h_{\infty}-u(r) .
$$

It follows easily from eqs. (44)-(45) that

$$
\nabla \Phi=-\tan \delta(r) \mathbf{r}+\mathbf{z}, \quad|\nabla \Phi|=\sec \delta(r) .
$$

Next, we will determine each of the energy density proposed in eq. (4) and, except for the $K_{1}^{a}$-term, its corresponding linearized version. We will show hereafter that the linearized version of the energy density in eq. (4) coincides with the one proposed by May (eq. (3) in May's manuscript, ${ }^{47}$ page 19). We note that

$$
\nabla \cdot \mathbf{a}=-\frac{\sin \delta(r)}{r}-\cos \delta(r) \delta^{\prime}(r)
$$

and

$$
\nabla \cdot \mathbf{n}=-\frac{\sin \theta(r)}{r}-\cos \theta(r) \theta^{\prime}(r) .
$$

Then the $K_{1}^{a}$-term in eq. (4) takes the form

$$
\frac{1}{2} K_{1}^{a}(\nabla \cdot \mathbf{a})^{2}=\frac{1}{2} K_{1}^{a}\left(\frac{\sin \delta(r)}{r}+\cos \delta(r) \delta^{\prime}(r)\right)^{2}
$$

while the $K_{1}^{n}$-term becomes

$$
\begin{aligned}
& \frac{1}{2} K_{1}^{n}\left(\nabla \cdot \mathbf{n}-s_{0}\right)^{2}= \\
& \frac{1}{2} K_{1}^{n}\left(\frac{\sin \theta(r)}{r}+\cos \theta(r) \theta^{\prime}(r)+s_{0}\right)^{2} .
\end{aligned}
$$

The above $K_{1}^{n}$-term can be approximated to the first order in $\theta$ by

$$
\frac{1}{2} K_{1}^{n}\left(\nabla \cdot \mathbf{n}-s_{0}\right)^{2} \doteqdot \frac{1}{2} K_{1}^{n}\left(\frac{\theta(r)}{r}+\theta^{\prime}(r)+s_{0}\right)^{2} .
$$

Let us consider the saddle-splay term in eq. (4) and recall that

$$
\begin{aligned}
& \frac{1}{2} K_{2} \nabla \cdot[(\mathbf{n} \cdot \nabla) \mathbf{n}-(\nabla \cdot \mathbf{n}) \mathbf{n}]= \\
& \frac{1}{2} K_{2}\left[\operatorname{tr}\left((\nabla \mathbf{n})^{2}\right)-(\nabla \cdot \mathbf{n})^{2}\right] .
\end{aligned}
$$

With the specific choice of $\mathbf{n}$ presented in eq. (41), it can be easily shown that

$$
\begin{gathered}
\operatorname{tr}\left((\nabla \mathbf{n})^{2}\right)=(\cos \theta(r))^{2}\left(\theta^{\prime}(r)\right)^{2}+\frac{(\sin \theta(r))^{2}}{r^{2}}, \\
(\nabla \cdot \mathbf{n})^{2}=\frac{(\sin \theta(r))^{2}}{r^{2}}+(\cos \theta(r))^{2}\left(\theta^{\prime}(r)\right)^{2} \\
+\frac{2}{r} \sin \theta(r) \cos \theta(r) \theta^{\prime}(r),
\end{gathered}
$$


from which it follows that

$$
\begin{aligned}
& \frac{1}{2} K_{2} \nabla \cdot[(\mathbf{n} \cdot \nabla) \mathbf{n}-(\nabla \cdot \mathbf{n}) \mathbf{n}]= \\
&-K_{2} \frac{\sin \theta(r) \cos \theta(r) \theta^{\prime}(r)}{r}
\end{aligned}
$$

The $K_{2}$-term in eq. (4) can be approximated to the first order in $\theta$ by

$$
\frac{1}{2} K_{2} \nabla \cdot[(\mathbf{n} \cdot \nabla) \mathbf{n}-(\nabla \cdot \mathbf{n}) \mathbf{n}] \doteqdot-K_{2} \frac{\theta(r) \theta^{\prime}(r)}{r}
$$

We now consider the compression-expansion term in eq. (4), which has been described in more detail in Sec. 3. By using eq. (47) we have that

$$
|\nabla \Phi|^{-1}(|\nabla \Phi|-1)=1-\cos \delta(r) .
$$

and, hence, the $B_{0}$-term takes the form

$$
\frac{1}{2} B_{0}|\nabla \Phi|^{-1}(|\nabla \Phi|-1)=\frac{1}{2} B_{0}(1-\cos \delta(r))^{2}
$$

The above expression can be rewritten using May's notation as

$$
|\nabla \Phi|^{-1}(|\nabla \Phi|-1)=1-\frac{h(\bar{r})}{h_{\infty}},
$$

where $h_{\infty}$ is the reference interlayer distance which remains unchanged away from the inclusion and $h(\bar{r})=b(r) \cos \theta(r)$ is the current interlayer distance as defined in Fig. 3. Moreover, we note that $h_{\infty}=b_{\infty}$ where $b_{\infty}$ represents the length of the lipid molecule in the reference configuration or away from the inclusion. Given eq. (60) and the previous remarks, the $B_{0^{-}}$ term in eq. (4) can be written as

$$
\frac{1}{2} B_{0}|\nabla \Phi|^{-2}(|\nabla \Phi|-1)^{2}=\frac{1}{2} B_{0}\left(1-\frac{b(r) \cos \theta(r)}{b_{\infty}}\right)^{2}
$$

which can be approximated to the first order in $\theta$ by

$$
\frac{1}{2} B_{0}|\nabla \Phi|^{-2}(|\nabla \Phi|-1)^{2} \doteqdot \frac{1}{2} B_{0}\left(1-\frac{b(r)}{b_{\infty}}\right)^{2}
$$

The coupling term in eq. (4) with $\mathbf{a}$ and $\mathbf{n}$ defined in eq. (40) and eq. (41), respectively, takes the form

$$
\begin{array}{r}
\frac{1}{2} B_{1}\left[1-(\mathbf{n} \cdot \mathbf{a})^{2}\right]=\frac{1}{2} B_{1}\left(1-\cos ^{2}(\theta(r)-\delta(r))\right)= \\
\frac{1}{2} B_{1} \sin ^{2}(\theta(r)-\delta(r)),
\end{array}
$$

which can be approximated to the first order in $\delta$ and $\theta$ to

$$
\frac{1}{2} B_{1}\left[1-(\mathbf{n} \cdot \mathbf{a})^{2}\right] \doteqdot \frac{1}{2} B_{1}(\theta(r)-\delta(r))^{2}
$$

The function $\delta(r)$ presented in eq. (39) can be then approximated to the first order in $\theta, \theta^{\prime}, b$, and $b^{\prime}$ to

$$
\delta(r) \doteqdot \arctan \left(b^{\prime}(r)\right) \doteqdot b^{\prime}(r)
$$

and, therefore, eq. (64) in terms of $b^{\prime}(r)$ becomes

$$
\frac{1}{2} B_{1}\left[1-(\mathbf{n} \cdot \mathbf{a})^{2}\right] \doteqdot \frac{1}{2} B_{1}\left(\theta(r)-b^{\prime}(r)\right)^{2} .
$$

Finally, by using eqs. (49) and (58), the $B_{2}$-term in eq. (4) can be expressed as

$$
\begin{aligned}
& B_{2}(\nabla \cdot \mathbf{n})\left(1-|\nabla \Phi|^{-1}\right)= \\
& \quad B_{2}\left(-\frac{\sin \theta(r)}{r}-\cos \theta(r) \theta^{\prime}(r)\right)(1-\cos \delta(r)) .
\end{aligned}
$$

In May's notation, the above term can be approximated to the first order in $\theta$ to

$$
\begin{aligned}
B_{2}(\nabla \cdot \mathbf{n})\left(1-|\nabla \Phi|^{-1}\right) & \doteqdot \\
& B_{2}\left(\frac{\theta(r)}{r}+\theta^{\prime}(r)\right)\left(\frac{b(r)}{b_{\infty}}-1\right) .
\end{aligned}
$$

The sum of the approximated terms presented in eqs. (52), (57), (62), (66), and (68) gives the energy density, $w_{M}$, presented by $\mathrm{May}^{47}$ for a planar lipid bilayer with a cylindrical inclusion:

$$
\begin{array}{r}
w_{M}=\frac{1}{2} K_{1}^{n}\left(\frac{\theta(r)}{r}+\theta^{\prime}(r)+s_{0}\right)^{2}-K_{2} \frac{\theta(r) \theta^{\prime}(r)}{r} \\
+\frac{1}{2} B_{0}\left(1-\frac{b(r)}{b_{0}}\right)^{2}+\frac{1}{2} B_{1}\left(\theta(r)-b^{\prime}(r)\right)^{2} \\
+B_{2}\left(\frac{\theta(r)}{r}+\theta^{\prime}(r)\right)\left(\frac{b(r)}{b_{0}}-1\right) .
\end{array}
$$

It must be noted that the material parameters are denoted differently in the energy presented by May: ${ }^{47} \kappa=K_{1}^{n}, \bar{k}=-K_{2}$, $K=B_{0}, \rho=B_{2}, k_{t}=B_{1}$.

By means of eqs. (50), (51), (56), (61), (63), (67), the energy density in eq. (4) can be written as

$$
\begin{aligned}
w_{D S}= & \frac{1}{2} K_{1}^{a}\left(\frac{\sin \delta(r)}{r}+\cos \delta(r) \delta^{\prime}(r)\right)^{2} \\
& +\frac{1}{2} K_{1}^{n}\left(\frac{\sin \theta(r)}{r}+\cos \theta(r) \theta^{\prime}(r)+s_{0}\right)^{2} \\
- & K_{2} \frac{\sin \theta(r) \cos \theta(r) \theta^{\prime}(r)}{r}+\frac{1}{2} B_{0}(1-\cos \delta(r))^{2} \\
+\frac{1}{2} B_{1} \sin ^{2}(\theta(r)-\delta(r)) & \\
- & B_{2}\left(\frac{\sin \theta(r)}{r}+\cos \theta(r) \theta^{\prime}(r)\right)(1-\cos \delta(r)) .
\end{aligned}
$$


In the next section, we will focus on solving the boundary value problems that are derived from the energy density (69) used by May ${ }^{47}$ and the energy density (70) proposed here. For the planar lipid bilayer considered hereafter, $s_{0}=0$ in eqs. (69)-(70).

\subsection{Boundary Value Problems}

The total energy, $W$, for the planar lipid bilayers depicted in Fig. 3(a) is

$$
W=\int_{\mathscr{V}} w d \mathscr{V}
$$

where $\mathscr{V}$ is the volume of the planar lipid bilayer and $w=w_{M}\left(r, \theta(r), \theta^{\prime}(r), b(r), b^{\prime}(r)\right)$ or $\quad w=$ $w_{D S}\left(r, \theta(r), \theta^{\prime}(r), \delta(r), \delta^{\prime}(r)\right)$. The equilibrium equations can be determined by minimizing the above total energy using variational methods. ${ }^{31}$ Since $\theta=\theta(r), \delta=\delta(r)$ and $b=b(r)$, the minimization of eq. (71) can be reduced via symmetry to the minimization of the total energy, $\tilde{W}$, defined as

$$
\tilde{W}=\int_{\tilde{V}} w d \tilde{\mathscr{V}}
$$

where $\tilde{V}$ is the section of lipid bilayer shown in Fig. 3(b). It can be easily shown that

$$
\tilde{W}=\frac{\pi}{2} \int_{R}^{\infty} d r \int_{0}^{h(r)} r w d z
$$

By following May's approach, ${ }^{47} h(r)=\frac{h_{R}+h_{\infty}}{2}$ is assumed to be constant so that the above integral can be approximated by

$$
\tilde{W} \approx \frac{\pi\left(h_{R}+h_{\infty}\right)}{4} \int_{R}^{\infty} r w d r .
$$

It is convenient to set $\hat{w}=r w$. A necessary condition for the total energy $\tilde{W}$ to have an extremum is that the EulerLagrange equations are satisfied. ${ }^{31}$ Thus, for $\hat{w}=r w_{M}$,

$$
\frac{\partial \hat{w}}{\partial \theta}-\frac{d}{d r}\left(\frac{\partial \hat{w}}{\partial \theta^{\prime}}\right)=0, \quad \frac{\partial \hat{w}}{\partial b}-\frac{d}{d r}\left(\frac{\partial \hat{w}}{\partial b^{\prime}}\right)=0,
$$

while, for $\hat{w}=r w_{D S}$,

$$
\frac{\partial \hat{w}}{\partial \theta}-\frac{d}{d r}\left(\frac{\partial \hat{w}}{\partial \theta^{\prime}}\right)=0, \quad \frac{\partial \hat{w}}{\partial \delta}-\frac{d}{d r}\left(\frac{\partial \hat{w}}{\partial \delta^{\prime}}\right)=0 .
$$

The Euler-Lagrange equations for the energy density $w=w_{M}$ are the following:

$$
\begin{aligned}
2 B_{1} b_{\infty} r^{2}\left(\theta-b^{\prime}\right)-K_{1}^{n} b_{\infty}\left(r^{2} \theta^{\prime \prime}\right. & \left.+r \theta^{\prime}-\theta\right) \\
& -B_{2} r^{2} b^{\prime}=0, \\
b_{\infty} B_{2}\left(r \theta^{\prime}+\theta\right)+2 B_{1} b_{\infty}^{2}\left[\theta-b^{\prime}\right. & \left.+r\left(\theta^{\prime}-b^{\prime \prime}\right)\right] \\
& +B_{0} r\left(b-b_{\infty}\right)=0,
\end{aligned}
$$

while, for the energy density $w=w_{D S}$, they are:

$$
\begin{aligned}
& K_{1}^{n} \cos \theta\left[\left(1+r^{2} \theta^{\prime 2}\right) \sin \theta\right.\left.-r\left(\theta^{\prime}+r \theta^{\prime \prime}\right) \cos \theta\right] \\
&-B_{1} r^{2} \sin (-\theta+\delta) \cos (-\theta+\delta) \\
&+B_{2} r^{2} \delta^{\prime} \cos \theta \sin \delta=0
\end{aligned}
$$

$$
\begin{aligned}
& K_{1}^{a} \cos \delta\left[\left(1+\delta^{\prime 2} r^{2}\right) \sin \delta-r\left(\delta^{\prime}+r \delta^{\prime \prime}\right) \cos \delta\right] \\
& +B_{1} r^{2} \sin (-\theta+\delta) \cos (-\theta+\delta)+B_{0} r^{2}(1-\cos \delta) \sin \delta \\
& \quad-B_{2} r\left(\sin \theta+r \theta^{\prime} \cos \theta\right) \sin \delta=0
\end{aligned}
$$

We impose the following boundary conditions to solve the system of eqs. (77)-(78):

$$
\begin{gathered}
\theta(R)=\frac{\pi}{6} \mathrm{rad}, \\
\theta^{\prime}(\infty)=0, \\
b(\infty)=2.5 \times 10^{-9} \mathrm{~m}, \\
b^{\prime}(\infty)=0 .
\end{gathered}
$$

The condition $\theta(R)=\frac{\pi}{6}$ rad is typical for a smectic A liquid crystals. ${ }^{27}$ The solution functions $\theta=\theta(r)$ and $b=b(r)$ (and their derivatives) can thus be computed by solving eqs. (77)(78) with the above boundary conditions. In May's formulation, once these functions are determined, the function $h=h(r)$, which defines the height of the lipid bilayer, and the function $\delta=\delta(r)$, which defines the normal to the layer, can be obtained from eqs. (36)-(37) and eq. (39), respectively. From eq. (39) one can compute the value of $\delta(r)$ at the inclusion, where $r=R$, and the value of $\delta^{\prime}(r)$ away from the inclusion, where $r=\infty$. These values are found to be

$$
\begin{gathered}
\delta(R)=0.72 \mathrm{rad} \\
\delta^{\prime}(\infty)=0
\end{gathered}
$$

The solution functions of the Euler-Lagrange equations derived from $w_{D S}$ are determined using the boundary conditions given in eqs. (81)-(82) and using the values of $\delta(R)$ and $\delta^{\prime}(\infty)$ in eqs. (85)-(86) computed as previously described. This procedure is employed in order to match the boundary conditions of the two systems of differential equations, eqs. (77)-(78) and eqs. (79)-(80), which have different sets of unknown functions. In our formulation, after determining the solution functions, $\delta(r)$ and $\theta(r)$, the height of the lipid bilayer is computed using eqs. (45)-(46). 


\begin{tabular}{cc}
\hline Material Parameter & Numerical Value \\
\hline \hline$K_{1}^{a}$ & $5 \times 10^{-12} \mathrm{~N}$ \\
$K_{1}^{n}$ & $5 \times 10^{-12} \mathrm{~N}$ \\
$K_{2}$ & $4 \times 10^{-12} \mathrm{~N}$ \\
$B_{0}$ & $8 \times 10^{5} \mathrm{Nm}^{-2}$ \\
$B_{1}$ & $8 \times 10^{5} \mathrm{Nm}^{-2}$ \\
$B_{2}$ & $10^{-7} \mathrm{Nm}^{-1}$ \\
\hline
\end{tabular}

Table 1 Material Parameters: $K_{1}^{a}, K_{1}^{n}$ and $K_{2}$ are based on values for a typical nematic liquid crystal. ${ }^{63}$ The value of $B_{0}$ is consistent with the estimated given by Kléman and Parodi ${ }^{42}$ for a typical smectic A liquid crystal. The estimate for $B_{1}$ is based on the assumption that it may be comparable in magnitude to $B_{0} .{ }^{58}$ The constant $B_{2}$ is based on the inequalities in eq. 9.

\begin{tabular}{ccc}
\hline $\begin{array}{c}\text { Parameter } \\
\text { (De Vita and Stewart) }\end{array}$ & $\begin{array}{c}\text { Parameter } \\
\text { (May) }\end{array}$ & $\begin{array}{c}\text { Numerical } \\
\text { Value }\end{array}$ \\
\hline \hline$u(\infty)$ & none & $0 \mathrm{~m}$ \\
$h_{\infty}$ & $h_{\infty}$ & $5 \times 10^{-9} \mathrm{~m}$ \\
$R$ & $R$ & $1 \times 10^{-9} \mathrm{~m}$ \\
$\infty$ & $\infty$ & $1 \times 10^{-8} \mathrm{~m}$ \\
$\theta(R)$ & $\theta(R)$ & $\frac{\pi}{6} \mathrm{rad}$ \\
$\theta(\infty)$ & $\theta(\infty)$ & $0 \mathrm{rad}$ \\
$\delta(R)$ & none & $0.72 \mathrm{rad}$ \\
$\delta(\infty)$ & none & $0 \mathrm{rad}^{-9} \mathrm{~m}$ \\
none & $b(\infty)$ & $2.5 \times 10^{-1}$ \\
none & $b^{\prime}(\infty)$ & $0 \mathrm{rad} \mathrm{m}^{-1}$ \\
\hline
\end{tabular}

Table 2 Parameters used for the boundary value problems. Note that $\delta(R)$ was computed from eq. (39) after finding $\theta(R), \theta^{\prime}(R), b(R)$, $b^{\prime}(R)$ by solving the boundary value problem derived from May's energy density.

\section{Results}

The energy densities defined in eqs. (69) and (70) were compared by solving the equilibrium eqs. (77)-(78) with boundary conditions defined in eqs. (81), (82), (83), and (84), and the equilibrium eqs. (79)-(80) with boundary conditions defined in eqs. (81), (82), (85) and (86). These boundary value problems describe the equilibrium configurations of a planar lipid bilayer with a cylindrical inclusion as depicted in Fig. 3(a). The values of the parameters in the energy densities were fixed to those listed in Table 1 while the geometrical dimensions of the system and boundary conditions are listed in Table 2. The coupled systems of nonlinear ordinary differential equations were solved numerically in Maple 12 (Maplesoft Inc.) by using the solver dsolve with a midpoint algorithm midrich method.

The solution function $\theta=\theta(r)$, which defines the tilt of the lipid molecules inside the planar lipid bilayer, is presented in
Fig. 4. As expected, the angle $\theta$, which is set to be $\frac{\pi}{6} \mathrm{rad}$ at $R=1 \mathrm{~nm}$ decreases as the radius $r$ increases and reaches zero as $r$ grows relatively large at around $10 \mathrm{~nm}$. It can be clearly seen in Fig. 4 that the tilt of the lipid molecules predicted using the proposed energy density is more pronounced than the one predicted using May's energy density away from the inclusion. Moreover, in May's predictions, the tilt of the lipid molecules becomes zero more rapidly as $r$ goes to $\infty$.

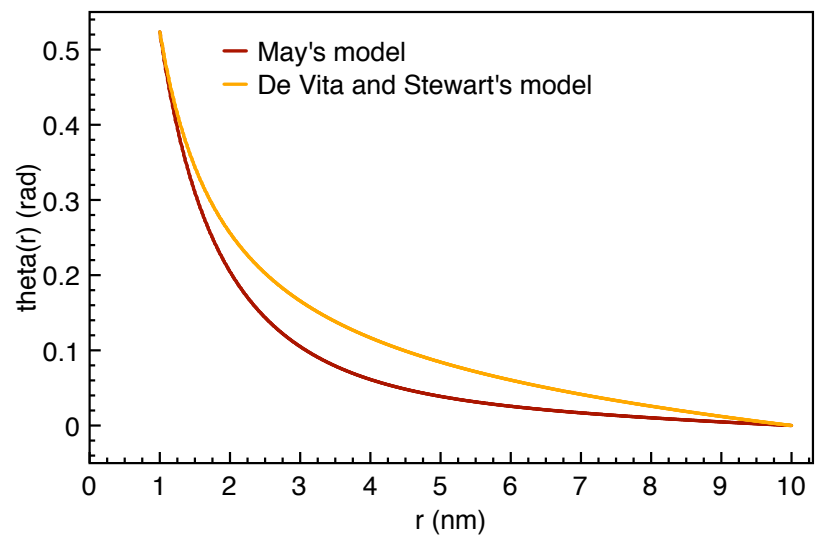

Fig. 4 Solution function $\theta=\theta(r)$ determined by solving the boundary valued problems derived from the newly proposed energy density in eq. (70) and the linearized energy density in eq. (69).

The solution function $\delta=\delta(r)$ of the boundary value problem derived from the newly proposed energy density is shown in Fig. 5. This function defines the normal to the lipid bilayer and, in our formulation, characterizes the displacement of the lipid layer via eq. (45). Although $\delta(r)$ is not directly computed as a solution of the boundary value problem derived from May's energy density, it can be easily computed from eq. (39) once the solution functions, $\theta(r), \theta^{\prime}(r), b(r)$, $b^{\prime}(r)$, are obtained. The value of $\delta(r)$ decreases as the radius increases according to both models. It can be noted that, when the radius of the bilayer becomes greater than $2 \mathrm{~nm}$, the difference between the values of $\delta(r)$ determined by the two boundary value problems is significant. The results show that, by considering the fully nonlinear terms in the energy density, greater changes in the normal to the lipid layer are predicted.

The height of the lipid bilayer can also be determined via eq. eqs. (36)-(37) in May's model and eqs. (45)-(46) in the proposed model (Fig. 6). As expected, the height is found to decrease close to the inclusion where $r=1 \mathrm{~nm}$. In particular, the height of the lipid bilayer is shown to decrease more for the boundary value problem derived from the proposed energy density. This is expected since, in the proposed formulation, the deformations are not assumed to be small.

The effect of various parameters or conditions on the solution of the boundary value problem derived from the proposed 


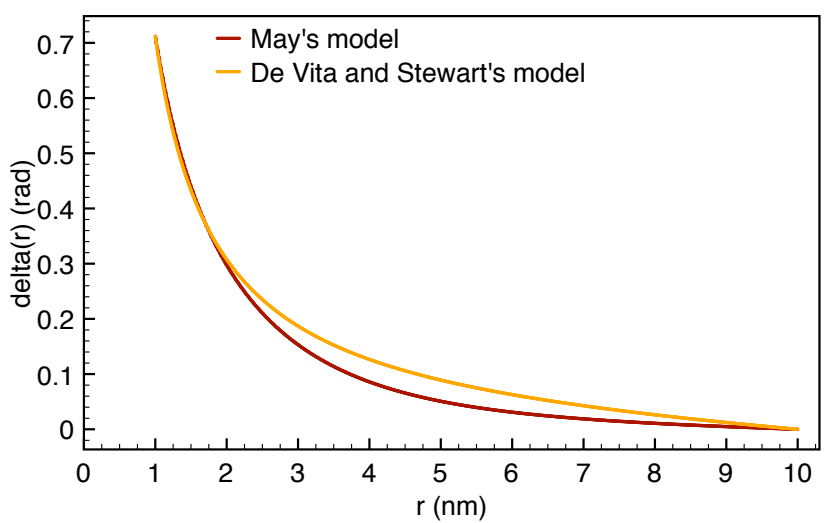

Fig. 5 Solution function $\delta=\delta(r)$ determined by solving the boundary valued problems derived from the newly proposed energy density in eq. (70) and the linearized energy density in eq. (69).

energy density can be also analyzed. For example, in Fig. 7 the height of the lipid bilayers at the inclusion, $h(R)$, is plotted as a function of the angle $\theta(R)$ that determines the orientation of the lipid molecules. The relationship between $h(R)$ and $\theta(R)$ appears to be nonlinear and, as $\theta(R)$ increases, $h(R)$ decreases.

\section{Discussion}

A new energy density was proposed to describe the equilibrium configurations of lipid bilayers. The energy accounts for the smectic A liquid crystalline structure of lipid bilayers, the decoupling between the layer normal and director, and large deformations. Analogies between the compression-expansion term of liquid crystals and lipid bilayers were also presented in detail. The proposed theoretical framework was tested by solving the boundary value problem derived when studying the deformation of a planar lipid bilayer induced by a cylindrical inclusion. The solution was directly compared with the solution of an equivalent boundary value problem obtained from a linearized version of the energy that was used in an earlier study by May. ${ }^{47}$ The deformations of the layer and distortions of the molecules were larger than those found using May's approach.

There has been much relatively recent activity on smectics and lipid bilayers which has attempted to deploy various versions of appropriate energy densities. The search for a rigorous and detailed approach that can be quite general has been the objective of this present article. Consideration is given for a separation between the usual director and layer normal, directions that are presumed coincident in the classical literature. ${ }^{24}$ The coupling between the deformations of lipid layers and lipid molecules has been considered by several au-

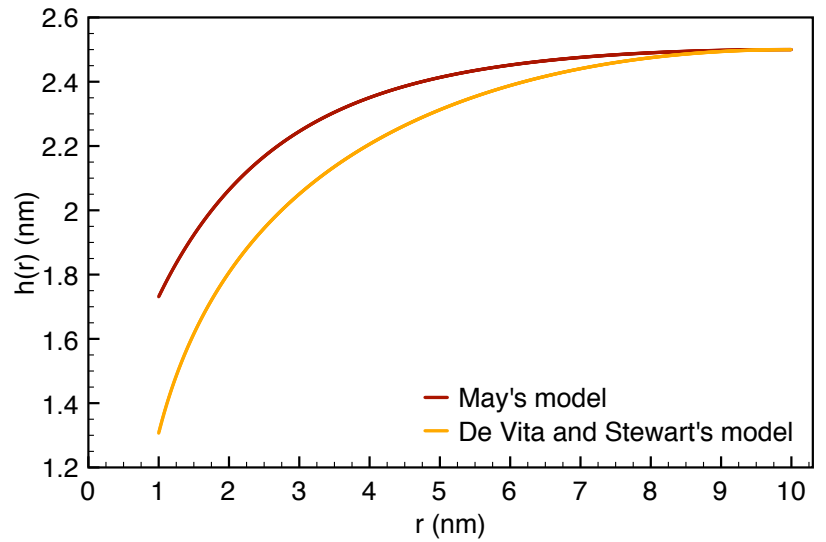

Fig. 6 The height of the bilayer, $h(r)$, determined by solving the BVP derived from eq. (70) (De Vita and Stewart's model) and eq. (69) (May's model) versus the radius $r$.

thors. ${ }^{30,34,44,47,48}$ These authors have, however, limited their analyses to small deformations and neglected some terms in the energy density to derive boundary value problems that are mathematically tractable. The results presented in Sec. 5 demonstrated the applicability of the proposed energy density and allowed comparisons with the earlier related work of others, including that by May. ${ }^{47}$

The energy density stated in eq. (4) has been derived from first principles in a systematic and general way. The contribution linked to $K_{1}^{n}$ was first considered rigorously by Frank ${ }^{28}$ in the context of 'splay' in the director alignment in general liquid crystals; the $K_{1}^{a}$-term is analogous to this in the context of the local lamellar layer normal. The expression for the $B_{0}$-term is a development from the work initiated by Kléman and Parodi ${ }^{42}$ and developed by Capriz, ${ }^{13,14}$ and Capriz and Napoli ${ }^{16}$ and Napoli. ${ }^{53,54}$ The contribution of the $B_{1}$-term originates from the concept of director tilt separating from the local layer normal, first discussed for smectic A liquid crystals by Ribotta and Durand. ${ }^{58}$ The interplay between compression and the separation of the director from the layer normal has been discussed in statics by Stewart ${ }^{64}$ and the present Authors, ${ }^{25,26}$ and by Stewart and Stewart ${ }^{65}$ in the context of an applied electric field. This separation has also been investigated in terms of basic flow problems by Auernhammer et al. ${ }^{3-5}$ Soddemann et al. ${ }^{62}$ and Stewart and Stewart. ${ }^{66}$ The $B_{2}-$ term is a generalized version of that first introduced by May. ${ }^{47}$

The analogies between the compression-expansion terms for lamellar systems and smectic A liquid crystals were presented. We remark that the results in eqs. (19) and (20) (with their analogues in eqs. (31) and (32)) were mentioned by Kléman and Parodi ${ }^{42}$ for the specific case of smectic A liquid crystals. These authors approximated the compression- 


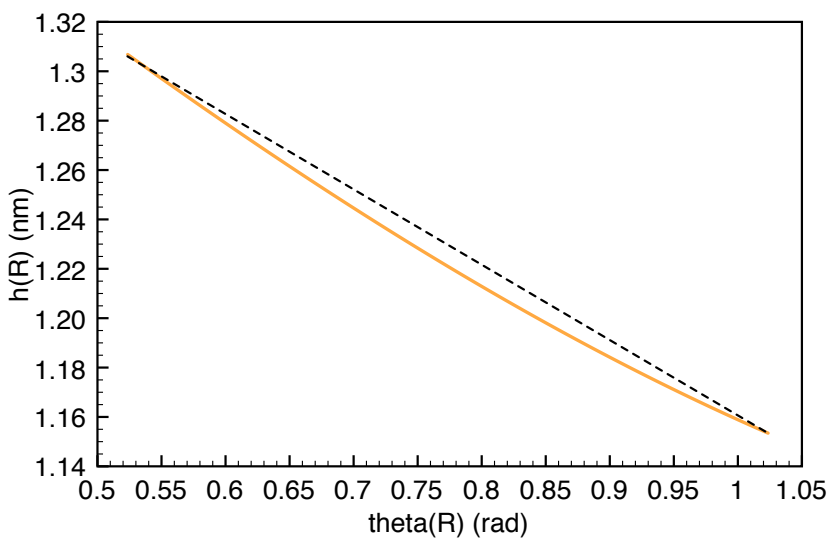

Fig. 7 The height of the bilayer, $h(R)$, versus the angle, $\theta(R)$, formed by the director with the z-axis at the inclusion where $r=R$ (orange line). The nonlinearities are better appreciated graphically when compared with a line (black dotted line).

expansion energy density by assuming small deformations. Thus, eq. (20) was replaced by $\varepsilon_{1} \equiv 1-|\nabla \Phi|$, an approximation that was introduced by Bidaux et al. ${ }^{7}$ and widely used throughout the literature thereafter; it is linearly equivalent in modulus to $\varepsilon \equiv 1-|\nabla \Phi|^{-1}$ for small gradients. An alternate strain can also be introduced as $\varepsilon_{2} \equiv\left(1-|\nabla \Phi|^{2}\right) / 2$, as considered by Kamien and Santangelo, ${ }^{41}$ who investigated its applications in Kamien et al. ${ }^{40}$ To linear order in the lamellar displacement, these three energy densities have the same modulus and lead to the same results; to see this, one can simply express $\varepsilon$ and $\varepsilon_{2}$ in terms of $\varepsilon_{1}$ via the substitution $|\nabla \Phi|=1-\varepsilon_{1}$ and expand to first order in $\varepsilon_{1} \cdot{ }^{42}$ The differences among $\varepsilon$, $\varepsilon_{1}, \varepsilon_{2}$ are only being apparent when nonlinear terms are included. Nevertheless, the forms in eqs. (20) and (32) that arise from the relations (19) and (31) have a natural interpretation: the magnitude of the gradient of $\Phi$ is largest when the level surfaces are closest together, as should be expected of level sets. This is perhaps the main reason why Capriz, ${ }^{13}$ Capriz and Napoli ${ }^{16}$ and Napoli ${ }^{54}$ adopted $\varepsilon$.

Some comments are in order regarding the values adopted for the material parameters in Table 1, some of which are based on well-established experimental values for lamellar smectic phases while others are estimates obtained via the inequalities in eq. (9). In the absence of specific experimental values for the elastic constants $K_{1}^{a}$ and $K_{1}^{n}$ for lipid bilayers, the values used here are typical for smectic materials. It is well known from the work on biaxial smectic $\mathrm{C}$ phases ${ }^{17,21,63}$ that these constants, which are expected to have comparable magnitudes, ${ }^{4}$ are similar to the Frank splay constant $K_{1}$ arising in general uniaxial liquid crystals. It has been calculated that $K_{1} \sim 5 \times 10^{-12}$ via a typical classical Helfrich-Hurault instability, ${ }^{63}$ as quoted in Table 1 . The elastic constant $K_{2}$ is the familiar saddle-splay constant that arises from the elastic theory of nematic liquid crystals and has been selected to satisfy, in conjunction with $K_{1}^{n}$ and $K_{1}^{a}$, the a priori estimates in eq. (9). The compression constant $B_{0}$ has been estimated for many smectic A systems to be of order $10^{6} \mathrm{Nm}^{-2}, 42$ in line with the adopted value in Table 1 . The coupling constant $B_{1}$ is relatively novel and was first introduced by Ribotta and Durand $^{58}$ and developed further by Oswald and Ben-Abraham, ${ }^{56}$ Auernhammer et al. ${ }^{3,4}$ and Soddemann et al.; ${ }^{62}$ it has been estimated from energetic considerations ${ }^{58}$ that $B_{1}$ should be comparable to $B_{0}$ in magnitude or smaller and this has been taken into account for the estimate in Table 1. The value of $B_{2}$ stated in Table 1 has been chosen to satisfy the inequalities in eq. (9). Both $B_{1}$ and $B_{2}$ have not been experimentally confirmed with any great accuracy, but an experiment to determine $B_{1}$ has been suggested ${ }^{65}$ and the influence of $B_{1}$ upon shear flow in smectic A has been investigated theoretically. ${ }^{3,62,66}$ The work presented in this article will hopefully encourage the design and development of directly relevant experiments for the measurement of the material parameters in lipid bilayers and related lamellar systems. It is through experimental data that the physical significance of such parameters can be identified.

One limitation of this and other studies ${ }^{47}$ is in the assumption that the height of the layer, $h(r)$, as the limit of integration in eq. (73), has a constant average value, here set to be $\frac{h_{R}+h_{\infty}}{2}$. This assumption was made to simplify the derivation of the boundary value problems via variational methods but it is likely that this approach introduces some error. The height of the lipid layer, $h(r)$, can be computed through eq. (37) in May's formulation and eq. (46) in our formulation only after finding the solution functions of the corresponding boundary value problems.

The energy density presented in eq. (4) will no doubt form the basis for more extensive discussions and investigations on smectics and lipid bilayers in complex geometries. It can be applied to describe different geometrical configurations of lipid bilayers under various loading scenarios and boundary conditions and allow more intricate analyses of dynamics. ${ }^{64}$ Depending on the specific problem tackled, other energetic contributions will need to be included such as, for example, surface tension and anchoring. ${ }^{25,26}$

The validity of the developed theoretical framework needs to be verified through experiments on lipid bilayers in which both the distortions of the layers and the lipid molecules can be quantified at molecular length scales. These challenging experiments may require the development of new methods in X-ray microscopy. Nevertheless, despite the lack of direct validation with experimental data, our continuum model thus far appears to be promising: it provides results that are, qualitatively, in agreements with molecular dynamics ${ }^{68}$ and coarse 
grained simulations. ${ }^{71}$

\section{References}

1 O. S. Andersen and R. E. Koeppe. Bilayer thickness and membrane protein function: an energetic perspective. Annu. Rev. Biophys. Biomol. Struct., 36:107-130, 2007.

2 A. Agrawal and D. J. Steigmann. Modeling protein-mediated morphology in biomembranes. Biomech. Model. Mechanobio., 8:371-379, 2009.

3 G. K. Auernhammer, H. R. Brand and H. Pleiner. The undulation instability in layered systems under shear flow - a simple model. Rheol. Acta, 39:215-222, 2000.

4 G. K. Auernhammer, H. R. Brand, and H. Pleiner. Shear-induced instabilities in layered liquids. Phys. Rev. E, 66:061707, 2002.

5 G. K. Auernhammer, H. R. Brand, and H. Pleiner. Erratum: Shearinduced instabilities in layered liquids. Phys. Rev. E, 71:049901, 2005.

6 G. V. Betangeri, S. A. Jenkins, and D. L. Parsons. Liposome Drug Delivery Systems. Technomic Publishing Company, 1993.

7 R. Bidaux, N. Boccara, G. Sarma, L. De Seze, P. G. De Gennes, and O. Parodi. Statistical properties of focal conic textures in smectic liquid crystals. J. de Physique, 34:661-672, 1973.

8 P. Biscari and R. Rosso. Inclusions embedded in lipid membranes. $J$. Phys. A: Math. Gen., 34:439-460, 2001.

9 P. Biscari and G. Napoli. Inclusion-induced boundary layers in lipid vesicles. Biomech. Model. Mechanobio., 6:297-301, 2007.

10 G. I. Blake and E. G. Virga. On the equilibrium of smectic C liquid crystals. Cont. Mech. Thermodyn., 8:323-339, 1996.

11 G. Brannigan, L. C. L. Lin, and F. L. H. Brown. Implicit solvent simulation models for biomembranes. Eur. Biophys. J., 35:104-124, 2006.

12 F. L. H. Brown. Elastic modeling of biomembranes and lipid bilayers. Annu. Rev. Phys. Chem., 59:685-712, 2008.

13 G. Capriz. Smectic liquid crystals as continua with latent microstructure. Meccanica, 30:621-627, 1995.

14 G. Capriz. Smectic elasticity. In R. C. Batra and M. F. Beatty, editors, Contemporary Research in the Mechanics and Mathematics of Materials, pages 199-204. CIMNE, Barcelona, 1996.

15 P. B. Canham. The minimum energy of bending as a possible explanation of the biconcave shape of the human red blood cell. J. Theor. Biol., 26:61, 1970.

16 G. Capriz and G. Napoli. Swelling and tilting in smectic layers. Appl. Math. Lett., 14:673-678, 2001.

17 T. Carlsson, I. W. Stewart, and F. M. Leslie. Theoretical studies of smectic C liquid crystals confined in a wedge: Stability considerations and Frederiks transitions. Liq. Cryst., 9, 661-678, 1991.

18 P. M. Chaikin and T. C. Lubensky. Principles of condensed matter physics. Cambridge University Press, Cambridge, 1995.

19 D. Collin, J. L. Gallani, and P. Martinoty. Abnormal sound damping in the smectic-C phase of terephthal-bis- $p$ - $p^{\prime}$-butylaniline (TBBA): Evidence for anharmonic effects. Phys. Rev. Lett., 58:254-257, 1987.

20 I. R. Cooke and M. Deserno. Coupling between lipid shape and membrane curvature. Biophy. J., 91:487-495, 2006.

21 I. Dahl and S. T. Lagerwall. Elastic and flexoelectric properties of chiral smectic-C phase and symmetry considerations on ferroelectric liquidcrystal cells. Ferroelectrics, 58, 215-243, 1984.

22 N. Dan, P. Pincus, and S. A. Safran. Membrane-induced interactions between inclusions. Langmuir, 9:2768-2771, 1993.

23 S. Daniel, F. Albertonio, and P. S. Cremer. Making lipid membranes rough, tough, and ready to hit the road. MRS Bull., 31:536-540, 2006.

24 P. G. de Gennes and J. Prost. The Physics of Liquid Crystals. Oxford University Press, Oxford, second edition, 1993.

25 R. De Vita and I. W. Stewart. Influence of weak anchoring upon the align- ment of smectic A liquid crystals with surface pretilt. J. Phys.: Condens. Matter, 20: 335101, 2008.

26 R. De Vita and I. W. Stewart. Nonlinearities in tilt and layer displacements of planar lipid bilayers. Eur. Phys. J. B, 32: 319-326, 2010.

27 S. J. Elston. The alignment of a liquid crystal in the smectic A phase in a high surface tilt cell. Liq. Cryst., 16:151-157, 1994.

28 F. C. Frank. I. Liquid crystals. On the theory of liquid crystals. Discuss. Faraday Soc., 25:19-28, 1958.

29 S. E. Feller. Molecular dynamics simulations of lipid bilayers. Curr. Opin. Colloid Interface Sci., 5:217-223, 2000.

30 J. B. Fournier. Microscopic membrane elasticity and interactions among membrane inclusions: interplay between the shape, dilation, tilt and tiltdifference modes. Eur. Phys. J. B, 11:261-272, 1999.

31 I. M. Gelfand and S. V. Fomin, Calculus of variations. Dover 2000

32 T. Gil, J. H. Ipsen, O. G. Mouritsen, M. C. Sabra, M. M. Sperotto, and M. J. Zuckermann. Theoretical analysis of protein organization in lipid membranes. Biochim. Biophys. Acta Rev. Biomembr., 1376:245-266, 1998.

33 K. M. Halverson, R. G. Panchal, T. L. Nguyen, R. Gussio, S. F. Little, M. Misakian, S. Bavari, and J. J. Kasianowicz. Anthrax biosensor: Protective antigen ion channel asymmetric blockade. J. of Biol. Chem., 280:34056-34062, 2005

34 M. Hamm and M. M. Kozlov. Elastic energy of tilt and bending of fluid membranes. Eur. Phys. J. E, 3:323-335, 2000.

35 W. Helfrich. Capillary flow of cholesteric and smectic liquid crystals. Phys. Rev. Lett., 23:372-374, 1969.

36 W. Helfrich. Elastic properties of lipid bilayers: Theory and possible experiments. Z. Naturforsch., 28:693-703, 1973.

37 I. Ivnitski, E. Wilkins, H. T. Tien, and A. Ottova. Electro-chemical biosensor based on supported planar lipid bilayers for fast detection of pathogenic bacteria. Electrochem. Commun., 2:457-460, 2000.

38 S. Izvekov and G. A. Voth. Multiscale coarse-graining of mixed phospholipid/cholesterol bilayers. J. Chem. Theory Comput., 2:637-648, 2006.

39 E. Jakobsson. Computer simulation studies of biological membranes: progress, promise and pitfalls. Trends Biochem. Sci., 22:339-344, 1997.

40 R. D. Kamien, D. R. Nelson, C. D. Santangelo, and V. Vitelli. Extrinsic curvature, geometric optics, and lamellar order on curved substrates. Phys. Rev. E, 80, 2009.

41 R. D. Kamien and C. D. Santangelo. Smectic liquid crystals: materials with one-dimensional, periodic order. Geom. Dedicata, 120:229-240, 2006.

42 M. Kléman and O. Parodi. Covariant elasticity for smectics A. J. de Physique, 36:671-681, 1975.

43 M. Kléman and O. D. Lavrentovich. Soft Matter Physics: An Introduction. Springer Verlag, 2003.

44 P. I. Kuzmin, S. A. Akimov, Y. A. Chizmadzhev, J. Zimmerberg, F.S. Cohen. Line tension and interaction energies of membrane rafts calculated from lipid splay and tilt. Biophys. J., 88:1120-1133, 2005.

45 F. C. MacKintosh and T. C. Lubensky. Orientational order, topology, and vesicle shapes. Phys. Rev. Lett., 67: 1169-1172, 1991

46 J. C. Maxwell. On hills and dales. Phil. Mag., 40:421-427, 1870.

47 S. May. Protein-induced bilayer deformations: the lipid tilt degree of freedom. Eur. Biophys. J., 29:17-28, 2000.

48 S. May, Y. Kozlovsky, A. Ben-Shaul, and M. M. Kozlov. Tilt modulus of a lipid monolayer. Eur. Phys. J. E, 14:299-308, 2004.

49 E. R. May, A. Narang, and D. I. Kopelevich. Role of molecular tilt in thermal fluctuations of lipid membranes. Phys. Rev. E, 76:21913, 2007.

50 L. Mirsky. An Introduction to Linear Algebra. Oxford University Press, Oxford, 1955.

51 M. Müller, K. Katsov, and M. Schick. Biological and synthetic membranes: What can be learned from a coarse-grained description? Phys. Rep., 434(5-6):113-176, 2006. 
52 T. Murtola, E. Falck, M. Patra, M. Karttunen, and I. Vattulainen. Coarsegrained model for phospholipid/cholesterol bilayer. J. Chem. Phys., 121:9156, 2004.

53 G. Napoli. Smectic liquid crystals with compressible elastic layers. Meccanica, 34:251-258, 1999.

54 G. Napoli. On smectic-A liquid crystals in an electrostatic field. IMA J. Appl. Math., 71:34-46, 2006.

55 Z. C. Ou-Yang, J. X. Liu, and Y. Z. Xie. Geometric Methods in the Elastic Theory of Membranes in Liquid Crystal Phases. World Scientific, Singapore, 1999.

56 P. Oswald and S. I. Ben-Abraham, Undulation instability under shear in smectic A liquid crystals. J. de Physique, 43, 1193-1197, 1982.

57 U. Raviv, D. J. Needleman, Y. Li, H. P. Miller, L. Wilson, and C. R. Safinya. Cationic liposome - microtubule complexes: Pathways to the formation of two state lipid-protein nanotubes with open or closed ends. Proc. Natl. Acad. Sci., 102:11167-11172, 2005.

58 R. Ribotta and G. Durand. Mechanical instabilities of smectic-A liquid crystals under dilative or compressive stresses J. de Physique, 38:179204, 1977.

59 C. D. Santangelo and R. D. Kamien. Elliptic phases: A study of the nonlinear elasticity of twist-grain boundaries. Phys. Rev. Lett., 96:137801, 2006.

60 U. Seifert. Configurations of fluid membranes and vesicles. Adv. Phys., 46:13-137, 1997.

61 N. Shahidzadeh, D. Bonn, O. Aguerre-Chariol, and J. Meunier. Large deformations of giant floppy vesicles in shear flow Phys. Rev. Lett., 81, 4268-4271, 1998.

62 T. Soddemann, G. K. Auernhammer, H. Guo, B. Dünweg, and K. Kremer. Shear-induced undulation of smectic-A: Molecular dynamics simulations vs. analytical theory. Eur. Phys. J. E, 13:141-151, 2004.

63 I. W. Stewart. The Static and Dynamic Continuum Theory of Liquid Crystals. Taylor and Francis, London and New York, 2004.

64 I. W. Stewart. Dynamic theory for smectic A liquid crystals. Continuиm Mech. Therm., 18:343-360, 2007.

65 F. Stewart and I. W. Stewart. A novel method for measuring compression constants in smectics. Mol. Cryst. Liq. Cryst., 478: 23-32, 2007.

66 I. W. Stewart and F. Stewart. Shear flow in smectic A liquid crystals. $J$. Phys.: Condens. Matter, 21: 465101, 2009.

67 S. Tristram-Nagle, R. Zhang, R. M. Suter, C. R. Worthington, W. J. Sun, and J. F. Nagle. Measurement of chain tilt angle in fully hydrated bilayers of gel phase lecithins. Biophys. J., 64:1097-1109, 1993.

68 M. Venturoli, B. Smit, M. M. Sperotto. Simulation studies of proteininduced bilayer deformations, and lipid-induced protein tilting, on a mesoscopic model for lipid bilayers with embedded proteins. Biophys J., 88:1778-1798, 2005.

69 A. J. Walker. The alignment of cylindrically layered smectic A liquid crystals with director tilt on the boundaries. J. Phys. A: Math. Theor, 41:385205, 2008.

70 J. R. L. Webb. Functions of Several Variables. Ellis Horwood, London, 1991.

71 B. West, F. L. Brown, F. Schmid. Membrane-protein interactions in a generic coarse-grained model for lipid bilayers. Biophys J. 96:101-115, 2009.

14 | Journal Name, 2010, [vol], 1-14 